\title{
Investigating partnerships and funding for the Physics Education Research community
}

\author{
Rebecca Rosenblatt \\ AAAS Science and Technology Policy Fellow, 2415 Eisenhower Ave., Alexandria, VA 22314 and \\ Tiliadal STEM Education: Solutions for Higher Education
}

This study will inform the Physics Education Research (PER) community about patterns of partnerships and collaborations between authors and patterns of funding sources for the PER community over the last ten years. The study involves a text-based analysis of all Physics Education Research Conference (PERC) proceedings between 2010 to 2019 to identify funding sources and to determine patterns. PERC proceedings were selected given the central role of PERC to the PER community. PERC proceedings represent the community across scope of projects from small to large, across stage of projects from beginning to finished, and across investigator experience from new to established researchers. Findings are contrasted with those from the Learning Sciences community to provide context for understanding the significance of patterns. The goal of this work is to provide insight into the community's history and ten-year trajectory and thus enable the community to move forward in new and better directions.

\footnotetext{
2020 PERC Proceedings edited by Wolf, Bennett, and Frank; Peer-reviewed, doi.org/10.1119/perc.2020.pr.Rosenblatt Published by the American Association of Physics Teachers under a Creative Commons Attribution 4.0 license. Further distribution must maintain the cover page and attribution to the article's authors.
} 


\section{INTRODUCTION}

This current study speaks to the Physics Education Research Conference (PERC) 2020 theme (Insights, Reflections, \& Future Directions: Emergent Themes in the Evolving PER Community) by investigating the history of funding, and funding streams, for contributed research projects presented in the PERC proceedings between 2010-2019. These funding data are also contrasted with those from the proceedings for the Computer-Supported Collaborative Learning (CSCL) and the International Conference on Learning Science (ICLS) proceedings to provide context for these funding results [1]. Additionally, this paper adds to the research literature on patterns in research partnerships/collaborations in PER by analyzing the author data from these contributed PERC proceedings. This research complements a study that focused on researcher collaborations from the American Journal of Physics, PhysRev-PER, and PERC from 1980-2010 [2].

Analyzing historical publication data is useful because recent shifts in policies and funding streams toward interdisciplinarity have included a focus on the workforce of the future [3]. Similarly, there is increased recognition by government funding agencies of the need to invest in partnerships and collaborations to achieve future-looking goals [4-6]. The PER community is uniquely placed to capitalize on these funding shifts as it sits in the overlaps of education, learning science, and physics as well as many other disciplines such as psychology and cognitive science. However, there is complex and important feedback that occurs between policies set through funding streams and the research completed by research communities such as PER. Indeed, McKenney suggested a call to action for the Learning Sciences community to better communicate with policy makers to help the community achieve future-looking goals [7], and I suggest that the PER community should be likewise inspired. Understanding the history of funding, partnerships, collaborations, and their outcomes in the PER community will be useful in determining how, where, and which policies might provide the most benefit to the community. Finally, this research is even more significant given the recent upheaval due to SARS-CoV-2 as it provides a benchmark for future efforts to investigate how the community's collaborations and outcomes are affected.

Informing the community on the history of these funding streams and author partnerships will allow the community to observe the scope of publication and collaboration in the PER community, leverage points for existing partnerships to innovate PER, identify underutilized grant streams and streams that others have found lucrative, and identify gaps in partnerships. The overarching goal of this work is to provide insight into the community's history and ten-year trajectory so that the community can consider how to best move the field forward in new directions.

This research seeks to answer the guiding research question: What is the landscape of funding and collaboration in PER among those who are published in the contributed PERC proceedings, and how has this landscape changed in the last
10 years? In addition, the following questions about funding were identified: What are the agencies that support this research? Within these agencies, what funding streams or disciplines are represented? Data based on institution collaborations and partnerships as represented by co-authorship seeks to answer the questions: Which institutions publish in PERC? Do authors mostly collaborate with authors at their institution or with other institutions? Has the pattern of collaboration shown in PERC changed as the PER community has grown and become more established?

\section{METHODOLOGY}

To answer these research questions, a text-based analysis was conducted of the PERC, ICLS, and CSCL proceedings from the last ten years to find award numbers linked to funding programs. These proceedings are publicly available and free $[1,8]$. These proceedings were selected because of the central role PERC plays in the PER community and the similarities of the PER and Learning Sciences communities. Additionally, these proceedings represent these communities across a broadly inclusive set of factors such as institution, scope of project from small to large, stage of project from beginning to finished projects, and experience of researchers from new to established in the field. However, these articles are a subset of the researchers and research being done in these communities and this article is limited by how fairly these proceedings sample their communities.

For analyses of funding streams, all contributed PERC proceedings from 2010-2019 were analyzed and the Learning Science conferences from 2010-2018. Since ICLS and CSCL proceedings are published every other year, six CSCL proceedings, five ICLS proceedings, and ten PERC proceedings were included. All information about the grant, or grants, that supported each paper's work were scraped using an advanced lexicon search within MAXQDA(C). Steps include the following: locating on past conference webpages all free digital (.pdf) volumes for the conference proceedings; searching for case sensitive "Acknowledgment(s)" and "Acknowledgement(s)"; using a regular expression (regex) pattern matching within an R Script to pull out digits for awards funded by the U.S. National Science Foundation (NSF); open coding of funding programs for other award sources outside of NSF; pulling data from NSF awards using the NSF Public Award Database; and cleaning the data to link NSF program data.

Author collaborations for PERC were analyzed by pulling the starting 250 words of each article, and open coding was used to extract the title, author names, and institutional affiliations. Author names were cleaned to match identical people who used different formats for naming themselves. This was done where it was clear that the persons were the same. For example, Joe T. Smith, Joseph Smith, and J.T. Smith at "Excellent University Alpha" were all considered the same person. However, if a different last name was used, the code counted these as two authors even though they may be the 
TABLE I. Funding Acknowledged in Contributed PERC Papers from 2010-2019. \% of Papers Funded = \# w/ Funding/ \# of Papers; $\%$ Papers Funded by NSF = \# with NSF Funding/ \# of Papers; \% of Funding by NSF = \# w/ NSF Funding/ \# w/ Funding.

\begin{tabular}{|c|c|c|c|c|c|c|c|c|c|c|}
\hline Year & 2010 & 2011 & 2012 & 2013 & 2014 & 2015 & 2016 & 2017 & 2018 & 2019 \\
\hline \# of Papers & 67 & 72 & 95 & 84 & 70 & 93 & 97 & 115 & 113 & 112 \\
\hline \# w/ Acknowledgments & 57 & 61 & 75 & 67 & 54 & 78 & 71 & 91 & 96 & 95 \\
\hline \# w/ Funding & 50 & 52 & 62 & 58 & 42 & 66 & 53 & 73 & 81 & 75 \\
\hline \# w/ NSF Funding & 35 & 41 & 51 & 46 & 32 & 49 & 45 & 59 & 62 & 61 \\
\hline \# of Unique NSF Funds & 31 & 53 & 44 & 46 & 44 & 50 & 52 & 56 & 61 & 62 \\
\hline$\%$ of Papers Funded & $74 \%$ & $72 \%$ & $65 \%$ & $69 \%$ & $60 \%$ & $71 \%$ & $54 \%$ & $63 \%$ & $72 \%$ & $67 \%$ \\
\hline$\%$ of Papers Funded by NSF & $56 \%$ & $60 \%$ & $56 \%$ & $55 \%$ & $46 \%$ & $53 \%$ & $46 \%$ & $51 \%$ & $55 \%$ & $54 \%$ \\
\hline$\%$ of Funding by NSF & $76 \%$ & $83 \%$ & $85 \%$ & $79 \%$ & $76 \%$ & $76 \%$ & $85 \%$ & $81 \%$ & $77 \%$ & $81 \%$ \\
\hline
\end{tabular}

same person. Additionally, efforts were made to capture multiple institution collaborations on a paper while giving each author a single affiliation for each publication.

\section{RESULTS AND DISCUSSION: FUNDING ANALYSIS}

Table I and Figure 1 present some of the main findings from these preliminary analyses. There were 918 contributed papers published in PERC proceedings from 2010-2019. 745 had acknowledgement sections or acknowledgments in the last few sentences of the article. 612 , or $67 \%$ of papers, acknowledged some source of funding. 489 papers, or 53\%, acknowledged U.S. National Science Foundation (NSF) funding with 481 giving the seven-digit number for that funding. Additionally, $80 \%$ of the papers that acknowledged funding credited NSF as one of the funding sources (see Table I). The community is also funded by institutional, local, and/or state grants as the next most common funding sources at 69 , or $11 \%$, of funding mentions, and there are small amounts of funding to the PER community from international sources such as Technological-140 grants (14 mentions) and 19 mentions of other granting institutions in various countries. Also, there are several foundations and businesses that provide a few grants that fund the community such as Howard Hughes Medical Institute (11 mentions), the Hewlett Foundation (9), the James S. McDonnell Foundation (7), and other sources.

In contrast, for the CSCL and ICLS proceedings from 2010-2018 there were 2,502 total submissions, with 1,062 of these having acknowledgments and 661 (or $26 \%$ of total papers) with funding. The funding for these conferences were more varied as they have a much larger and more international community, but still over $48 \%$ of funding acknowledged in CSCL and ICLS came from NSF. Additionally, approximately $60 \%$ of the funding came from U.S. sources and $40 \%$ from international sources. $16 \%$ of funding was from Europe with the European Union playing a large role in this funding, and 14\% was from Asia with the Japan Society for the Promotion of Science and the Ministry of Education in Singapore playing large roles. 54 mentions, or 8\%, of funding came from the U.S. Department of Education (US ED) for this community while only 8 mentions, or $1 \%$, of funding for PERC was from US ED. Additionally, CSCL and ICLS papers were funded by local, state, or university funds (62 mentions or $9 \%$ ), the Spencer Foundation (11 papers), the Gates Foundation (10), Google grants (5), and other sources.

Figure 1 shows funding by conference and NSF funding program. Note that ICLS has more than CSCL, which is consistent with this community's greater size. These graphs show that funding from NSF's Division of Research on Learning

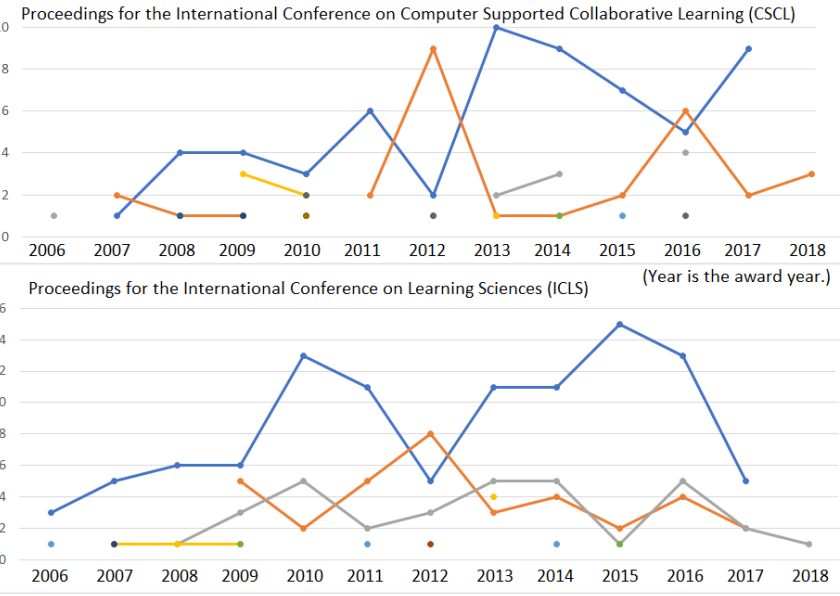

Proceedings for the Physics Education Research Conference (PERC) (*The yaxis is not the same across graphs.)

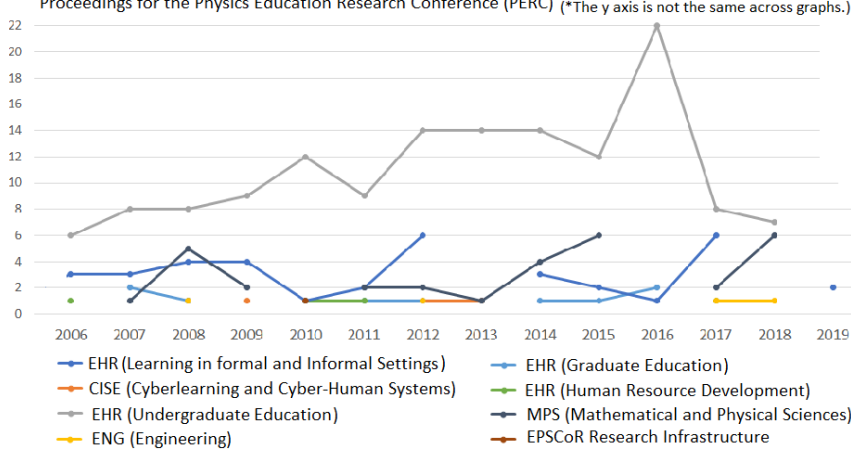

FIG. 1. Number of Awards Acknowledged from NSF Funding Programs for the CSCL, ICLS, and PERC proceedings 2010-2019. 
within the Education and Human Resources directorate (see the blue line in Fig. 1) has consistently funded both communities. The Computer and Information Sciences directorate (the orange line) has played a greater role in the CSCL community and Undergraduate Education (in grey) in the ICLS community. In contrast, the PER community is funded predominately by the Division of Undergraduate Education and the Directorate of Mathematical and Physical Science.

The Division of Research on Learning is commonly associated with research in K-12 and informal learning settings, and Computer and Information Sciences is commonly associated with Cyberlearning for Work at the Human-Technology Frontier respectively. Lower funding for PER from these sources suggests that these are either as yet untapped areas of research in PER and/or untapped areas of funding. Figure 1 also shows that some NSF programs may be funding sources that both of these communities could utilize more. For example, all three communities had relatively low numbers of grants from the Division of Graduate Education.

These funding similarities suggest overlaps in community policy interests. However, these two communities are different. Thus, interpreting the meaning of this funding data is difficult and inferences from this data should be carefully considered. Given the current dependence of PER funding on the National Science Foundation, expanding the range of funding sources might capitalize on the varied missions of funding agencies and dampen any effects from changes to federal budgets.

\section{RESULTS AND DISCUSSION: COLLABORATIONS}

As the PER community moves forward into new and interesting innovations, it is useful to reflect on the partnerships and collaborations between PER researchers and the outcomes of these partnerships. In an effort to establish the number and kinds of partnerships that the PER community has, the author data from the contributed PERC proceeding (2010-2019) were analyzed for the number of authors who were collaborating on papers, whether these collaborations were multi-institutional or single institution, and which institutions were co-publishing (see Table III).

There were 918 contributed papers included in the PERC proceedings from 2010-2019 [8]. 65 of these 918 papers $7 \%$ were single author and $853,93 \%$, were collaborations of multiple authors (See Table III). Of these collaborations, 64\% had all authors who were at the same institution, and $36 \%$ were collaborations where authors were from different institutions. Of the papers with multiple institutions, it was significantly more likely for a majority of authors to be from the same institution and only one of the authors to be from a different institution than for all authors to be from separate institutions. For example, for 4-author papers, half of the papers had authors from multiple institutions; however, about $60 \%$ of these are from two institution collaborations and not 3 or 4 institution collaborations. These data show that the PER community re- lies heavily on collaborations/partnerships to accomplish research goals. It also shows that most author collaborations happen via partnerships at a single institution, which suggests that intra-institution collaborations such as PER groups and/or graduate student to faculty partnerships are important for publication productivity. Lastly, it indicates that while the with-in institution collaborations are very important there are key cross-institution interactions that are driving research productivity as measured by authorship.

In addition, see Figure 2, multiple author partnerships are increasing with time as the community becomes more established and larger. Single-author papers stayed the same at around 6 papers a year over the 10 years as did two-author collaborations at around 25 a year but both 3 -author and 4 (or more)-author papers increased with time.

In terms of institutions that participated, 274 different locations were associated with 2,897 author instances. University of Colorado Boulder was the most represented with 331 author participations. University of Pittsburgh was next with 152. In total 19 institutions accounted for $57 \%$ (1,659 of the 2,897 instances) of author participation. These are universities with graduate PER programs, and it is not surprising that these institutions have high participation. However, noting the two institutions external to the U.S. and the geographic spread is interesting and may be useful for students looking for programs of study, job placements, and/or people searching for collaborators. Additionally, while these 19 institutions represent much of the participation in PERC, it is important

TABLE II. Institutions with the most authors for PERC 2010-2019.

\begin{tabular}{lcc}
\hline \hline Institution & Author Instances & Percentage \\
\hline University of Colorado Boulder & 331 & $11.4 \%$ \\
University of Pittsburgh & 152 & $5.2 \%$ \\
Michigan State University & 127 & $4.4 \%$ \\
Florida International & 123 & $4.2 \%$ \\
Kansas State University & 110 & $3.8 \%$ \\
University of Maryland & 90 & $3.1 \%$ \\
Oregon State University & 78 & $2.7 \%$ \\
University of Central Florida & 77 & $2.7 \%$ \\
The Ohio State University & 67 & $2.3 \%$ \\
Texas State University & 62 & $2.1 \%$ \\
Rochester Institute of Technology & 60 & $2.1 \%$ \\
University of Maine & 60 & $2.1 \%$ \\
University of Washington & 55 & $1.9 \%$ \\
Western Michigan University & 54 & $1.9 \%$ \\
Seattle Pacific University & 51 & $1.8 \%$ \\
Univ. of British Colombia (Canada) & 49 & $1.7 \%$ \\
California State University Chico & 40 & $1.4 \%$ \\
Tecnologico de Monterrey (Mexico) & 38 & $1.3 \%$ \\
University of Illinois & 35 & $1.2 \%$ \\
255 Additional Institutions & 1,238 & $42.7 \%$ \\
\hline \hline
\end{tabular}


TABLE III. Partnerships \& Collaborations from Contributed PERC papers from 2010-2019.

\begin{tabular}{|c|c|c|c|c|c|c|c|c|c|c|}
\hline Year & 1 Author & 2 Author & 3 Author & 4 Author & 5 Author & 6 Author & 7 Author & 8 Author & 9 Author & Instances of Author \\
\hline 2010 & 4 & 20 & 21 & 11 & 8 & 3 & 0 & 0 & 0 & 209 \\
\hline 2011 & 6 & 25 & 17 & 15 & 7 & 2 & 0 & 0 & 0 & 214 \\
\hline 2012 & 6 & 38 & 24 & 19 & 4 & 3 & 1 & 0 & 0 & 275 \\
\hline 2013 & 9 & 23 & 28 & 14 & 5 & 3 & 2 & 0 & 0 & 252 \\
\hline 2014 & 4 & 28 & 15 & 15 & 5 & 0 & 2 & 1 & 0 & 212 \\
\hline 2015 & 14 & 20 & 25 & 22 & 8 & 2 & 0 & 1 & 0 & 281 \\
\hline 2016 & 11 & 25 & 24 & 22 & 12 & 2 & 0 & 1 & 0 & 301 \\
\hline 2017 & 3 & 30 & 30 & 26 & 24 & 1 & 1 & 0 & 0 & 390 \\
\hline 2018 & 2 & 26 & 42 & 26 & 9 & 5 & 1 & 2 & 0 & 382 \\
\hline 2019 & 6 & 27 & 31 & 27 & 12 & 6 & 1 & 1 & 1 & 381 \\
\hline Total & 65 & 262 & 257 & 197 & 94 & 29 & 8 & 5 & 1 & 2,897 \\
\hline Single Inst. Papers & 65 & 209 & 175 & 100 & 32 & 8 & 0 & 2 & 0 & 591 \\
\hline Multi Inst. Papers & - & 53 & 82 & 97 & 62 & 21 & 8 & 3 & 1 & 327 \\
\hline \# of Unique Authors & 54 & 315 & 411 & 444 & 293 & 136 & 34 & 40 & 9 & 1,085 \\
\hline
\end{tabular}

The number of papers with multiple authors increased with time

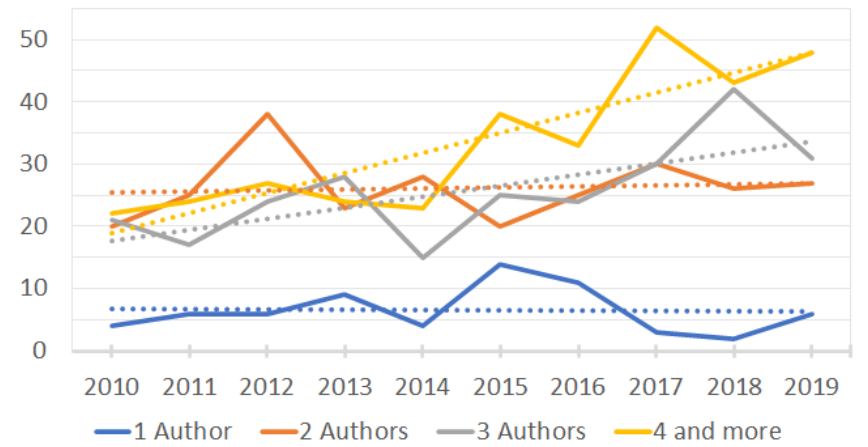

FIG. 2. Collaborations on Contributed PERC Papers

to note that there are 255 other places from which people are contributing. 128 of these have one or two contributions while 58 locations are averaging at least yearly participation.

\section{CONCLUSIONS}

To provide insight into the PER community's history and ten-year trajectory, a text-based analysis of all PERC proceedings between 2010 to 2019 is provided that identifies funding sources and determines patterns of author collaboration. PERC proceedings were selected given the central role of PERC to the PER community and its representation of the community across scope of project, stage of project, and researcher experience. The goal of this work is to enable the community to move forward in new and better directions.

It was observed that the work presented in PERC shows a much higher funding rate than the Learning Science commu- nity at $67 \%$ of papers acknowledging funding as compared to $26 \%$. NSF is by far the most common source of funding and within NSF the Division of Undergraduate Education and the Directorate of Mathematical and Physical Science are the most common sources. Comparing these funding sources with Learning Sciences, the Division of Research on Learning and CyberLearning and Cyber-Human Systems are the most common. It is also noted that both communities have few acknowledgments from Graduate Education. Outside of NSF, the PER community might consider US ED as a largely untapped resource since US ED accounts for only $1 \%$ of PER funding but $8 \%$ of Learning Science funding.

In addition, these data show that PER has a diverse community across the U.S. and abroad. There were 274 unique institutions from which 1,085 authors contributed work from 2010 to 2019. In addition, the field shows an increasing trend in author numbers, collaborative paper submissions, international participation, and numbers of papers being included in PERC proceedings. While the community is broad, it should be noted that much of the PERC authorship comes from large universities with Physics Education Research groups. This suggests these places are key to community engagement, but assuming that most of this research is on students attending these universities, this implies that the body of PERC research may not represent students who are taught at high schools, community colleges, and bachelor's only institutions [9].

Plans are in place to continue this analysis to connect research themes to these locations and to extend this data to other disciplines outside of PER and the Learning Sciences such as Engineering Education. Additionally, these author and grant data have been compiled to an interactive map by using an R script and a spreadsheet with paper titles, authors, year, grant information, and institutional zip code interpreted into latitude and longitude. The author is working on a permanent venue for hosting this data set and map. 
[1] https://www.isls.org/

[2] K. A. Anderson, M. Crespi, and E. C. Sayre Phys. Rev. Phys. Educ. Res. 13, 010121 (2017). DOI:10.1103/PhysRevPhysEducRes.13.010121

[3] NSF's $10 \quad$ Big Ideas. Retrieved from https://www.nsf.gov/news/special_reports/big_ideas/

[4] Sustainable Development Goals. Retrieved from https://sustainabledevelopment.un.org/

[5] Cyberlearning Community Report: The State of Cyberlearning and the Future of Learning with Technology. Menlo Park CA: SRI International. United Nations. (2019).
[6] J. Roschelle, W. Martin, J. Ahn, \& P. Schank, (Eds.) (2017) https://circlcenter.org/resources/community-report/

[7] S. McKenney, Journal of the Learning Sciences 27(1), 1-7 (2018). DOI: 10.1080/10508406.2017.1404404

[8] https://www.per-central.org/perc/Proceedings.cfm

[9] S. Kanim and X. Cid A(2020) Phys. Rev. Phys. Educ. Res. 16(2), 020106 (2020). DOI:10.1103/PhysRevPhysEducRes.16.020106 